\title{
A Peptide-Based Neoantigen Vaccine for Patients With Advanced Pancreatic Cancer Refractory to Standard Treatment
}

\section{Zheling Chen}

Zhejiang Provincial People's Hospital

\section{Shanshan Zhang}

Zhejiang University

\section{Ning Han}

Hangzhou Neoantigen Therapeutics Co.,Ltd.

\section{Jiahong Jiang}

Zhejiang Provincial People's Hospital

\section{Yunyun Xu}

Zhejiang Provincial People's Hospital

\section{Dongying Ma}

Hangzhou Neoantigen Therapeutics Co.,Ltd.

\section{Huimin Wang}

Hangzhou Neoantigen Therapeutics Co.,Ltd.

\section{Xiaojie Guo}

Hangzhou Neoantigen Therapeutics Co.,Ltd.

\section{Kai Luo}

Hangzhou Neoantigen Therapeutics Co.,Ltd.

\section{Min Qiu}

Hangzhou Neoantigen Therapeutics Co.,Ltd.

\section{Qinxue Huang}

Hangzhou Neoantigen Therapeutics Co.,Ltd.

\section{Liu Liang}

Hangzhou Neoantigen Therapeutics Co.,Ltd.

\section{Fan Mo}

\section{Zhejiang University}

\section{Shuqing Chen}

Zhejiang University

\section{Liu Yang ( $\square$ yangliuqq2003@163.com )}

Key laboratory of tumor molecular diagnosis and individualized medicine of zhejiang province,zhejiang provincial people's hospital,hangzhou,china 
Research

Keywords: Neoantigen, Pancreatic cancer, Vaccine, Immunotherapy

Posted Date: December 21st, 2020

DOl: https://doi.org/10.21203/rs.3.rs-130888/v1

License: (c) (i) This work is licensed under a Creative Commons Attribution 4.0 International License. Read Full License 


\section{Abstract}

Background: Tumor-specific neoantigens are considered as personalized and potential ultimate targets for cancer immunotherapy. Recently, neoantigen cancer vaccines have been designed to train the patient's immune system to specifically target and kill tumor cells. However, the safety and efficacy of neoantigen vaccines in pancreatic cancer treatment remain poorly understood.

Methods: Personalized peptide neoantigen cancer vaccines were successfully designed and manufactured for pancreatic cancers with low tumor mutation burden. Seven patients with advanced pancreatic cancer refractory to standard treatments were enrolled and treated with personalized peptide neoantigen vaccine, iNeo-Vac-P01. Besides the evaluation of the safety and clinical efficacy of iNeo-VacP01, pre- and post-vaccination peripheral blood samples were collected to analyze the vaccine's immunogenicity.

Results: For all 7 patients, none sever vaccine-related adverse effects was witnessed. The mean progression free survival, overall survival (OS) and OS associated with vaccine treatment were 3.1, 24.1 and 8.3 months, respectively. For Patient P01, who had a 21-month OS associated with vaccine treatment, the abundance of the TCR clone remarkably increased after vaccination, indicating the potential of iNeo-Vac-P01 to specifically induce a subset of T cells to kill tumor cells. This study also demonstrated that the quantity of IFN-y in peripheral blood might be a potential biomarker for OS.

Conclusions: We believed that it was the first tentative study focused in the application of peptide-based neoantigen cancer vaccine in advanced pancreatic cancer. Promisingly, personalized peptide neoantigen vaccine might provide a new strategy to improve the limited clinical efficacy for pancreatic cancer.

Trial registration: ClinicalTrials.gov, NCT03645148. Registered August 24, 2018 - Retrospectively registered, https://www.clinicaltrials.gov/ct2/show/NCT03645148?

term=neoantigen\&cond=Pancreatic+Cancer\&cntry $=\mathrm{CN} \& d r a w=2 \&$ rank $=1$

\section{Background}

As one of the deadliest cancers, pancreatic cancer has a five-year overall survival (OS) rate of only $9.3 \%$ (1). The poor prognosis of pancreatic cancer mainly results from the lack of screening and early detection strategies, the disease's propensity of early dissemination and relatively poor sensitivity to current standard treatments. Many patients are diagnosed with advanced tumors(2). Even for those initially diagnosed in a very early state and received standard treatments such as surgical resection combined with systemic radiotherapy or chemotherapy, their 5-year OS rate has not yet exceeded $25 \%(3)$. Meanwhile, the incidence of pancreatic cancer is increasing worldwide. According to the 2018 International Cancer Research Institute (IARC) GLOBOCAN statistics, there were 458,918 new cases and 432,242 deaths of pancreatic cancer, accounting for $2.5 \%$ of total new cancer cases and $4.5 \%$ of total deaths caused by all cancer types in 2018(4). Therefore, to resolve the contradiction between the lack of 
effective treatments for pancreatic cancer and its increasing incidence, greater efforts should be directed to the development of more promising therapies.

Recently, immunotherapy has demonstrated its attractive potential in the treatment of various solid tumors. The emergence of chimeric antigen receptor T cell immunotherapy (CAR-T) and immune checkpoint blocking antibodies such as anti-PD-1/PD-L1 has casted new light on cancer immunotherapy $(5,6)$. However, for pancreatic cancer, immune checkpoint inhibitors alone or combined with chemotherapy have not achieved evident positive outcomes in clinical studies $(7,8)$. On the other hand, over the past five years, several clinical trials have been launched to investigate the profiles of therapeutic neoantigen cancer vaccines including their safety, tolerability and efficacy. Neoantigens are mostly generated from non-synonymous mutations in cancer cells, therefore they are usually exempted from central tolerance. Personalized peptide neoantigen vaccines are designed to train a patient's immune system in order to target and kill tumor cells specifically through a series of steps: delivery of neoantigens to antigen presenting cells (APCs); presentation of tumor-specific neoantigens by APCs to T cells; and activation of cytotoxic T cells to recognize and eliminate tumor cells(9). Ideally, the activated tumor-specific cytotoxic T-lymphocytes could infiltrate into tumors, turning "cold" tumors into "hot" tumors, thereby leading to enhanced anticancer efficacy.

In 2017, two famous studies published by the research groups of Professors Wu and Sahin, respectively, have demonstrated that neoantigen vaccines based on peptides or RNA could not only induce significant regression of advanced melanoma, but also provide long-term protection against tumor relapse and metastasis $(10,11)$. Moreover, with the data on patients with newly diagnosed glioblastoma published in 2019 by the same groups respectively, the potential of personalized peptide neoantigen vaccines to elicit sustained T cell response and increase the number of tumor-infiltrating T cells was further confirmed(12). Recently, the results from a phase $\mathrm{lb}$ trial demonstrated feasibility, safety, and immunogenicity of the combination of personalized peptide neoantigen vaccines plus PD-1 inhibition in patients with advanced melanoma, non-small cell lung cancer, or bladder cancer(13). Other studies focusing on colon cancer(14) and esophageal cancer(15) have also confirmed the effectiveness of neoantigen vaccines. For pancreatic cancer, although the efficacy of tumor-associated antigen (TAA) peptide vaccine candidates has been investigated in several case reports or clinical studies of small sample sizes(16-18), the anticancer effects of personalized peptide neoantigen cancer vaccines have yet to be extensively tested.

Thus, we reported here the first tentative study of personalized peptide neoantigen cancer vaccine (iNeoVac-P01) in patients with advanced pancreatic cancer. The safety, tolerability, and anticancer efficacy of this vaccine were investigated through comprehensive analyses of patients' molecular and immune responses after vaccination. For some patients, to maximize their clinical benefits, iNeo-Vac-P01 was administered with concomitant medical therapies such as chemotherapy or immune checkpoint blockade. Our preliminary results not only demonstrated good safety and tolerability of iNeo-Vac-P01, but also indicated its potential to elicit specific $T$ cell activation and improve the survival duration of patients with advanced pancreatic cancer. 


\section{Methods}

\section{Patients}

Eligible patients were aged 18 years or older; with advanced pancreatic cancer confirmed histologically or cytologically, chemotherapy intolerance or disease progression after second-line treatments, at least one measurable lesion as per investigator-assessed Response Evaluation Criteria in Solid Tumors (RECIST; version 1.1), an Eastern Cooperative Oncology Group (ECOG) performance status of 0 or 1 , and good functioning major organs, such as heart, liver and kidney. All patients could provide sufficient tumor tissue and blood samples for whole exome sequencing (WES) and RNA sequencing (if fresh tumor tissue is available).

Key exclusion criteria included: having other malignant tumor except for cured basal cell carcinoma, thyroid carcinoma, or cervical dysplasia; lacking identified neoantigen by sequencing; having received bone marrow or stem cell transplant; allergic to any drugs, polypeptides, or other potential immunotherapies.

\section{Trial design and treatment}

This was a single-arm, open-label, investigator-initiated clinical study at Zhejiang Provincial People's Hospital in China (NCT03645148), with the primary endpoints of safety and feasibility, and secondary endpoints of efficacy evaluated through progression-free survival (PFS), overall survival (OS) and neoantigen-specific immune responses. The safety of the study was assessed on the basis of occurrence of adverse events (AEs). The feasibility of this trial was assessed by whether the neoantigens could be identified by our in-house pipeline iNeo-Suite and the synthesis of the peptide vaccines could be accomplished for clinical use.

iNeo-Vac-P01 for each patient comprises 5 20 peptides at lengths of 15 35 amino acids. The peptides were firstly grouped into 2 4 pools based on their HLA typing, affinity and allele frequency, and then injected subcutaneously (s.c.) in the upper arms and paraumbilical region respectively, at the dose of 100 $\mu \mathrm{g}$ per peptide. Thirty minutes prior to each injection of iNeo-Vac-P01, $40 \mu \mathrm{g}$ granulocyte-macrophage colony-stimulating factor (GM-CSF) was administered subcutaneously nearby the immunization site as adjuvant $(10,12,19-21)$. Patients were primed with iNeo-Vac-P01 on day 1, 4, 8, 15 and 22 (prime phase), and boosted with the same vaccine formulations on day 78 and 162 (boost phase). Additional booster shots might be applied depending on the ethics and the patients' potential benefits from doing so according to the clinical research protocol. Whether or not to apply concomitant medical therapies such as chemotherapy and immune checkpoint blockade was decided by clinicians according to each patient's clinical response. The whole treatment regimen of each patient was summarized in Table S1.

Clinical assessment, monitoring and follow-up of patients with advanced pancreatic cancer in this study were conducted, including physical examination such as ECOG performance, vital sign, blood test and 
urinalysis for the safety evaluation; imaging examination at baseline and post-vaccination for clinical efficacy assessment; IFN-y Enzyme-Linked Immunospot (ELISpot) Assay and flow cytometry (T cell subsets and cytokines) applied pre- and post-vaccination for the detection of specific immune response.

Tumors were assessed by investigators according to RECIST v1.1 criterion at baseline and approximately every 8 weeks thereafter. The clinical response of each patient was evaluated not only throughout, but also at a regular interval of 3 months after the whole vaccination regimen until disease progression, development of cumulative toxic effects or patient discontinuation. The occurrence and severity of adverse events (AEs) were recorded, and graded based on the National Cancer Institute Common Terminology Criteria for Adverse Events (version 4.0) throughout the treatment period.

The study protocol was approved by the Institutional Review Board and Independent Ethics Committee, and implemented in accordance with the Declaration of Helsinki and the International Conference on Harmonization Guidelines for Good Clinical Practice. All patients had signed informed consent forms before immunization.

\section{Generation of personalized peptide neoantigen vaccines}

To identify mutation-derived neoantigens, tumor tissues and blood samples were obtained from patients either directly after surgery, or by biopsy or intravenous blood sampling. Whole exome sequencing (WES) was conducted on these samples using Hiseq 4000 NGS platforms (Illumina) with coverage depths of 500x for tumor cells and 100x for blood cells (Novogene Biotech Co., Ltd., Beijing, China)(22-26). In particular, formalin-fixed paraffin-embedded (FFPE) samples were used for WES when fresh tumor samples were unavailable.

The bioinformatics analysis was performed by our in-house pipeline iNeo-Suite consisting of multiple modules: sequencing read filtering, genome alignment, mutation calling, HLA typing, MHC affinity prediction, gene expression profiling, vaccine peptide sequence design, and mutation centered prioritization based on therapeutic potency (Supplementary Methods).

To generate personalized peptide neoantigen cancer vaccine, iNeo-Vac-P01, customized clinical-grade long peptides were manufactured through chemical synthesis at GMP-like standard (bacteria-free, > $95.0 \%$ purity, and quantities of bacterial endotoxin less than $10 \mathrm{EU} / \mathrm{mg}$ ). The water solubility of these synthesized peptides was tested before excluding insoluble peptides from iNeo-Vac-P01 formulation.

\section{IFN-y Enzyme-Linked Immunospot (ELISpot) Assay}

To confirm the immunogenicity of iNeo-Vac-P01, ELISpot assays were performed for each patient at a series of timepoints pre- and post-vaccination. Peripheral blood $(10-30 \mathrm{~mL})$ was obtained from each patient for the isolation of peripheral blood mononuclear cells (PBMCs). PBMCs were then co-incubated ( $2 \times 10^{5}$ cells per well) with peptides for $16-24$ hours using Human IFN-y pre-coated ELISpot kit following 
the standard protocol. An automatic plate reader with appropriate parameters was used to count spots in ELISpot plate (Supplementary Methods).

\section{T cell receptor (TCR) sequencing}

To monitor the change of T cell population of each patient, T cell receptor (TCR) $\beta$ chains were sequenced before and after vaccination. RNA extraction of PBMCs was performed using RNeasy Plus Mini Kit (Qiagen). Samples were analyzed by High-throughput sequencing of TCR using ImmuHub TCR profiling system at a deep level (ImmuQuad Biotech). Briefly, a 5' RACE unbiased amplification protocol was used. Unique molecular identifiers (UMIs) introduced in the course of CDNA synthesis were used to control bottlenecks and eliminate the errors of PCR and sequencing. Sequencing was performed on an Illumina HiSeq system with PE150 mode (IIlumina). One common adaptor with UMI was added to the $5^{\prime}$ of cDNA during the synthesis of first-strand CDNA. One reverse primer corresponding to the constant (C) regions of each TCRa and $\beta$ was designed to facilitate PCR amplification of CDNA sequences in a less biased manner. The UMls attached to each raw sequence reads were applied for sequencing error correction and PCR duplication removal. V, D, J and C segments were mapped with IMGT. CDR3 regions were extracted, and clonotype assembled for all clones. The special nucleotide/amino acid sequences of CDR3 region of TCR $\beta$ subunit were determined. Those with out-of-frame or stop codon sequences were removed from the identified TCR $\beta$ repertoire. We further defined the amount of each TCR $\beta$ clonotype as the total number of TCR $\beta$ clones sharing the same nucleotide sequence of CDR3 region.

\section{Cytometric analysis of T-lymphocyte and cytometric bead array (CBA) analysis of cytokines}

To quantify the activation of T cells after vaccination, flow cytometry was applied to analyze the proportions of different types of $T$ cells extracted from patients' peripheral blood samples and labeled with several antibodies. To examine the cytokines secreted from activated T cells after vaccination, the concentrations of cytokines in patients' peripheral blood were measured by CBA, according to the manufacturer's protocol (Supplementary Methods).

\section{Statistical analysis}

Data from the patients received at least one dose of iNeo-Vac-P01 was analyzed for the assessment of safety and clinical efficacy. Descriptive statistics was applied to determine the characteristics of baseline, and assess the safety of iNeo-Vac-P01. The target lesions of each patient were measured before the treatment and then every two months during the treatment to monitor the changes in lesion sizes. Disease control rate (DCR) was defined as the proportion of patients who had complete response (CR), partial response (PR) and stable disease (SD) for best clinical response. Standard RECISTV1.1 guideline 
was applied for the analyses of all clinical data. The survival curves were plotted by GraphPad Prism 5 (v5.01).

\section{Results}

\section{Patients and demographics}

From January 1, 2018 to March 31, 2020, a total of 7 patients with advanced pancreatic cancer were enrolled in the trial to receive iNeo-Vac-P01. These patients had previously received standard chemotherapy or surgery, and experienced cancer relapse or metastasis. Table 1 listed the demographics of all 7 patients including their clinical baseline data. Six (85.71\%) patients had adenocarcinoma, and one (14.29\%) patient had squamous cell carcinoma. Four (57.14\%) patients had liver metastases, and three $(42.86 \%)$ patients had peritoneum metastases. In addition, four $(57.14 \%)$ patients had higher CA199 levels at baseline compared to the other three (42.86\%) patients with normal levels.

\section{Feasibility of preparation and application of neoantigen in patients with advanced pancreatic cancer}

The results of WES on both patient's tumor tissue and peripheral blood cells were shown in Supplementary Table S2. Neoantigens were predicted and prioritized using our in-house pipeline iNeoSuite, in consideration of allelic frequency of mutation, affinity between mutated peptide and HLA class I and II, as well as feasibility of peptide synthesis. The results were shown in Supplementary Tables S3, S4 and S5. Clinical-grade long peptides (15-35 amino acids) incorporating multiple neo-epitopes of both HLA class I and II were synthesized (Supplementary Table S4). The turn-around time of the whole process ranged from 1.5 to 3 months, depending on the qualities of tumor samples and the sequences of long peptides.

Despite of the lower tumor mutation burden (TMB) in pancreatic cancers, personalized long peptides were successfully manufactured for all seven patients, respectively, following the identification of sufficient neoantigens. Different from the clinical studies published before(10, 12),no organic solvent such as DMSO was applied to enhance the water solubility of personalized long peptides due to ethics concerns. Among the total 94 long peptides that were designed and synthesized successfully, seventy peptides with good water solubility were selected for patient immunizing (Supplementary Table S6). Most patients (5 out of 7) received vaccines with more than 10 peptides (Supplementary Table S4 and S6). There was a median of 12 long peptides immunized for each patient, which comprised a median of 9 class I neo-epitopes and 20 class II neo-epitopes per peptide.

\section{Treatment and follow-up of patients}


Patients were scheduled to receive iNeo-Vac-P01 together with GM-CSF as adjuvant (Fig. 1). The median follow-up duration for all patients was 9.7 months, ranging from 2 months to 21 months before the deadline March 31, 2020. All patients completed the prime phase of immunization (Fig 1). The average duration of treatment was 2.57 months, ranging from 1 month to 5 months. Patients P02, P03, P06 and P07 were assessed to have stable disease (SD) during vaccine treatment; Patients P01 and P04 displayed partial response during vaccine treatment, and had good disease control for a period of time after the whole vaccine treatment; while patients P05 had progressive disease (PD) in the boost phase of the peptide vaccine treatment.

\section{Safety and side effects}

During the vaccine treatment, none of the seven patients had grade 3-4 iNeo-Vac-P01-related adverse events defined by NCI CTCAE 4.03. One of the patients (P06) experienced a mild rash after vaccine injection but recovered within one week. In comparison, all seven patients experienced different degrees of adverse reactions after receiving chemotherapy. The most common serious adverse events of chemotherapy among these patients were hematological toxic events, including neutropenia (7/7) and anemia (5/7). Other chemotherapy-related adverse reactions including gastrointestinal reactions, rashes, and fever were displayed in Table 2.

\section{Clinical response}

RECIST 1.1 criteria was used to assess target lesions in all patients. The mean OS of the 7 patients was 24.1 months (11 months to 31.4 months), and the mean PFS was 3.1 months (Table 3 ). Calculated from the patient's first immunization, the mean OS associated with the peptide vaccine treatment was 8.3 months (3 months to 21 months). Except for Patient P04, the other six patients had died. The survival curve showed the OS of seven patients (Fig. 2A). Corresponding to the data shown in Table 3, the survival rate was about $50 \%$ at 24 months. Clinical response of patients in this study was showed in Fig. 2B. Three of the 7 patients displayed tumor reduction of target lesions. While Patients P01 and P04 were evaluated as PR, for their target lesions reduced by $54 \%$ and $57 \%$, respectively, compared with baseline; Patient P06 maintained stable disease with a target lesion reduced by only $5 \%$. On the other hand, although the other four patients had different degrees of increase in their target lesions during vaccine treatment; Patient P07, P02, and P03 were all assessed as SD with less than $20 \%$ increase of their target lesions. Only Patient P05 displayed PD with a 40\% increase in target lesion. The disease control rate (DCR) of the 7 patients was $85.71 \%$.

\section{Case report of Patient P01}

Patient P01 was first diagnosed with pancreatic cancer with liver metastasis in July 2017. From July 2017 to February 2018, she was given AG regimen (paclitaxel albumin plusS-1) for 8 cycles of first-line 
conversion chemotherapy. Under general anesthesia, laparoscopic radical pancreatectomy was performed on March 5th, 2018. No chemotherapy was given after surgery due to the patient's poor physical condition. In less than three months, lymph node metastasis was found. From 6th June, 2018 to 7th November 2018, Patient P01 received a total of 8 doses of iNeo-Vac-P01, including 5 prime and 3 boost vaccinations. Also, to maximize the patient's clinical benefits, 5-cycle AS second-line treatment was given from July 2018 to November 2018 as concomitant therapy. In February 2019, a tumor marker was detected while CT imaging showed no tumor recurrence. In response, the patient soon received 2-cycle AS systemic chemotherapy. After the treatment, the patient's disease was considered to be well controlled until bone metastasis and pleural effusion occurred in September 2019. Since then, the disease progressed rapidly. After supportive treatment and chemotherapy, Patient P01 died in March 2020. The OS and PFS of Patient P01 were 32 and 8 months, respectively. In particular, the OS associated with iNeoVac-P01 treatment was 21 months. The whole treatment regimen was shown in Fig. 3A. MRI images showed the nodule of pancreaticogastric space lesion regression at $4^{\text {th }}$ month after first immunization compared to that of the baseline level (Fig. 3B). No iNeo-Vac-P01-related serious AE was noted during the whole vaccine treatment. Ex vivo IFN- $y$ ELISpot of PBMCs confirmed robust de novo immune response against all neoantigen peptides after vaccination, with a peak at Week 3 (Fig. 3C and D). TCR sequencing of peripheral T cells revealed that the abundance of the TCR clone (CASSPGQGVYNEQFF) considerably increased after vaccination. Meanwhile, a new TCR clone (CASSLGTGYNEQFF) was detected after vaccination (Fig. 3E). These data suggested that a subset of $T$ cells with neoantigen specificities had been induced by iNeo-Vac-P01. The concomitant iNeo-Vac-P01 therapy and chemotherapy might have generated synergetic benefits to prolong the OS and PFS of Patient P01.

\section{Immune response}

Ex vivo IFN-y ELISpot assay was performed with autologous PBMCs after vaccination, demonstrating the potentials of iNeo-Vac-P01 to induce the activation of T cells in 5 out of 7 patients. For each patient, the number of IFN- $\gamma$ spots per $10^{5}$ PBMCs of the peptide or peptide pool with best response had been shown in Fig. 4. For patient P05 and P07, no evident response was found pre- and post-vaccination. Overall, 31 out of $70(44.3 \%)$ individual long peptides elicited measurable peptide-specific immune responses (positive results in ELISpot assay after vaccination). To identify the potential biomarkers for clinical response, several indexes including IL2, IL4, IL6, IL10, TNF-a, IFN-ץ were tested before and during vaccine treatment. For Patients P01, P02, and P07, the levels of IFN- $y$ in their peripheral blood samples increased after treatments (Supplementary Table S7). No statistical conclusion could be drawn from the data of IL2, IL4, IL6, IL10 and TNF-a. Moreover, for all 7 patients, the proportions of CD4+ CTLA4+ T cells and CD8+ CTLA4+ T cells in peripheral blood increased during vaccination phase (Fig.5). To be noted, not all of the patients could provide enough blood samples for analysis, mostly due to their poor conditions at the designated timepoints (such as P03 and P05).

\section{Discussion}


In this pilot study, the safety and well safety and tolerability of neoantigen peptide vaccine iNeo-Vac-P01 were verified in pancreatic cancer patients. Overall, only one patient showed iNeo-Vac-P01-related AE (slight rash), and recovered without any nursing. For all 7 patients, none SAE was witnessed during the whole iNeo-Vac-P01 treatment period.

Currently, the median overall survival time for patients with advanced pancreatic cancer is 6 to 9 months(27). Albumin paclitaxel combined with gemcitabine or mFOLFIRINOX regimen (5-fluorouracil [5FU], irinotecan and oxaliplatin) is recommended as the standard first-line treatment by the guidelines(28, 29). Meanwhile, there is no standard chemotherapy regimen after second-line treatment. A stratified analysis of the posterior survival of pancreatic cancer patients showed that the median survival time of patients with high-risk pancreatic cancer was only 1.4 months, and that of low-risk patients was less than 12 months(30). In comparison, our study showed not only the good safety and tolerability, but also the promising antitumor efficacy of neoantigen vaccine as a second-line or later treatment for pancreatic cancer patients. Generally speaking, patients with advanced pancreatic cancer have large tumor burdens. Therefore, instead of applying neoantigen vaccine monotherapy to all enrolled patients, P01 and P07 received vaccine treatments combined with chemotherapy or PD-1 inhibitor respectively, to maximize their clinical benefits. The details of patients' treatment regimen were listed in Supplementary Table S1. In our study, the mean OS and PFS of the pancreatic cancer patients were 24.1 and 3.1 months respectively, longer than all of the data reported in other clinical studies as listed previously. In particular, the mean OS associated with the iNeo-Vac-P01 treatment was 8.3 months. Although it is an exploratory study with a small sample size, the results have demonstrated the encouraging potentials of neoantigen vaccine as a second-line or later treatment to extend the survival duration of pancreatic cancer patients.

KRAS mutation is a common driver mutation for some cancer types including pancreatic cancer, and it is usually incorporated in the "shared" tumor antigen combinations as "off-the-shelf" tumor vaccines. In a previous study of patients with advanced cancer (pancreatic cancer, cholangiocarcinoma and colorectal cancer), Rahma et al. demonstrated that the patients could achieve a mean PFS and OS of 3.6 months and 16.9 months respectively, with an immune response rate of $54 \%$ when treated with the vaccine formulation incorporating KRAS G12D, G12V and G12C peptides(31). Although it is a promising strategy to treat the patients with KRAS mutation, the "off-the-shelf" tumor vaccines cannot fulfill the clinical needs for those patients who do not have the mutations. Different from the vaccines based on "shared" antigens, personalized peptide neoantigen cancer vaccines are customized for each patient. In this study, only 5 out of 7 patients had KRAS mutations (supplementary table S8). KRAS mutations were also included in the design of personalized iNeo-Vac-P01 for these 5 patients to maximize their clinical benefits. In comparison, Patient P01, one of the two patients without KRAS mutation, had a significant extension of survival duration as a result of the concomitant iNeo-Vac-P01 vaccine therapy and chemotherapy, as shown in Fig. 3 and Table S1.

It is also noteworthy that Patient P01 started the personalized peptide vaccination only three months after the primary tumor resection, leading to relatively lower tumor burden compared with other patients. The iNeo-Vac-P01-related OS of Patient P01 was 21 months, longer than that of any other patients in this 
study. More studies should be conducted in the future to explore whether a pancreatic cancer patient might achieve longer survival duration if neoantigen tumor vaccine is given when the tumor burden is low. Previously, a case report had described a pancreatic cancer patient who began SVN-2B peptide vaccine treatment during the adjuvant treatment stage(17). After the vaccine regimen, isolated lung metastases were observed in this patient but well controlled by surgery. It was reported that this patient had a survival duration of more than 10 years(17). In light of this case report together with the clinical findings of Patient P01, we believe that it is important to investigate the "perfect" timing of the administration of neoantigen tumor vaccine, for example, starting the vaccination regimen in the adjuvant treatment stage or when the tumor burden is low.

In addition, several T cell subsets and cytokines in peripheral blood were evaluated in this study to identify potential biomarkers for clinical response. The fact that the patients with relatively longer survival duration (P01, P02 and P07) had an increase of the secretion of IFN- $y$ after iNeo-Vac-P01 treatments, indicated the potentials of IFN- $\gamma$ in peripheral blood as a biomarker for overall survival duration. Also, both the proportions of CD4+ CTLA4+ T cells and CD8+ CTLA4+ T cells in peripheral blood increased in all 7 patients after vaccination, as a result of the inducement of peptide vaccines. Thus, the combination of personalized neoantigen peptide vaccination and anti-CTLA-4 antibody treatment might be promising for pancreatic cancer patients, which requires further studies with special considerations in the timing for vaccine administration to achieve maximized clinical benefits.

\section{Conclusions}

In a word, the preliminary results of this study have demonstrated both the feasibility of neoantigen selection for pancreatic cancer patient with low tumor mutation burden (TMB) and the good tolerability of the designed peptide vaccine, iNeo-Vac-P01 for pancreatic cancer patients. Our results were important and complementary to those of previous published studies in neoantigen cancer vaccines treating other types of cancers. The development of personalized peptide neoantigen cancer vaccines might provide a new strategy to improve the limited clinical efficacy of traditional treatments for pancreatic cancer.

\section{Declarations}

\section{Ethics approval and consent to participate}

The study was reviewed by the Ethics Committee of Zhejiang Provincial People's Hospital. The final study protocol was approved by institutional review board and independent ethics committee, and conducted in accordance with Declaration of Helsinki and the International Conference on Harmonisation Guidelines for Good Clinical Practice. All the patients included in the study have signed the informed consents.

\section{Consent for publication}


The patients have signed the consents and give their consents for the materialto be published. All the authors have given their consents for the materials about the patients to appear in a BMC publication.

\section{Availability of data and material}

All data generated or analyzed during this study are included in this published article.

\section{Competing interests}

1. Mo is an employee for Hangzhou Neoantigen Therapeutics Co., Ltd and Hangzhou Al-Force Therapeutics. S. Zhang, N. Han, D. Ma, H. Wang, X. Guo, K. Luo, M. Qiu, Q. Huang, L. Liang, S. Chen are employees for Hangzhou Neoantigen Therapeutics Co., Ltd. No potential conflicts of interest were disclosed by the other authors.

\section{Funding}

This work was supported by National Key Research and Development Program of China (2017YFC0908600 to Shuqing Chen), National Natural Science Foundation of China (81772575 to Liu Yang, 81972455 to Liu Yang and 81430081 to Zheling Chen).

\section{Authors' contributions}

Zheling Chen, Shanshan Zhang, Shuqing Chen, Liu Yang and Fan Mo contributed to the conception and design of the work. Zheling Chen, Ning Han, Dongying Ma and Xiaojie Guo performed the studies. Jiahong Jiang, Yunyun Xu, Huimin Wang, Kai Luo and Min Qiu contributed to data processing and analysis. Zheling Chen, Qinxue Huang, Fan Mo and Ning Han helped with the statistical analysis. Zheling Chen, Shanshan Zhang, Shuqing Chen and Liu Yang prepared the majority of the manuscript with Fan Mo, Ning Han revised it critically. All authors have given final approvals to the version to be published.

\section{Acknowledgements}

The authors thank all patients participated in the trial and their families, as well as Zhejiang Provincial People's Hospital as clinical site.

\section{References}

1. Idachaba S, Dada O, Abimbola O, Olayinka O, Uma A, Olunu E, et al. A Review of Pancreatic Cancer: Epidemiology, Genetics, Screening, and Management. Open access Macedonian journal of medical sciences. 2019;7(4):663-71. 
2. Bliss LA, Witkowski ER, Yang CJ, Tseng JF. Outcomes in operative management of pancreatic cancer. Journal of surgical oncology. 2014;110(5):592-8.

3. Vincent A, Herman J, Schulick R, Hruban RH, Goggins M. Pancreatic cancer. Lancet. 2011;378(9791):607-20.

4. Bray F, Ferlay J, Soerjomataram I, Siegel RL, Torre LA, Jemal A. Global cancer statistics 2018 : GLOBOCAN estimates of incidence and mortality worldwide for 36 cancers in 185 countries. CA: a cancer journal for clinicians. 2018;68(6):394-424.

5. Nair R, Westin J. CAR T-Cells. Advances in experimental medicine and biology. 2020;1244:215-33.

6. Han Y, Liu D, Li L. PD-1/PD-L1 pathway: current researches in cancer. American journal of cancer research. 2020;10(3):727-42.

7. Weiss GJ, Waypa J, Blaydorn L, Coats J, McGahey K, Sangal A, et al. A phase lb study of pembrolizumab plus chemotherapy in patients with advanced cancer (PembroPlus). British journal of cancer. 2017; 117(1):33-40.

8. Aglietta M, Barone C, Sawyer MB, Moore MJ, Miller WH, Jr., Bagala C, et al. A phase I dose escalation trial of tremelimumab $(\mathrm{CP}-675,206)$ in combination with gemcitabine in chemotherapy-naive patients with metastatic pancreatic cancer. Annals of oncology : official journal of the European Society for Medical Oncology. 2014;25(9):1750-5.

9. Yadav M, Jhunjhunwala S, Phung QT, Lupardus P, Tanguay J, Bumbaca S, et al. Predicting immunogenic tumour mutations by combining mass spectrometry and exome sequencing. Nature. 2014;515(7528):572-6.

10. Ott PA, Hu Z, Keskin DB, Shukla SA, Sun J, Bozym DJ, et al. An immunogenic personal neoantigen vaccine for patients with melanoma. Nature. 2017;547(7662):217-21.

11. Sahin U, Derhovanessian E, Miller M, Kloke BP, Simon P, Lower M, et al. Personalized RNA mutanome vaccines mobilize poly-specific therapeutic immunity against cancer. Nature. 2017;547(7662):222-6.

12. Keskin DB, Anandappa AJ, Sun J, Tirosh I, Mathewson ND, Li S, et al. Neoantigen vaccine generates intratumoral T cell responses in phase lb glioblastoma trial. Nature. 2019;565(7738):234-9.

13. Ott PA, Hu-Lieskovan S, Chmielowski B, Govindan R, Naing A, Bhardwaj N, et al. A Phase lb Trial of Personalized Neoantigen Therapy Plus Anti-PD-1 in Patients with Advanced Melanoma, Non-small Cell Lung Cancer, or Bladder Cancer. Cell. 2020;183(2):347-62 e24.

14. Kuai R, Ochyl LJ, Bahjat KS, Schwendeman A, Moon JJ. Designer vaccine nanodiscs for personalized cancer immunotherapy. Nature materials. 2017;16(4):489-96.

15. Forghanifard MM, Gholamin M, Moaven O, Farshchian M, Ghahraman M, Aledavood A, et al. Neoantigen in esophageal squamous cell carcinoma for dendritic cell-based cancer vaccine development. Medical oncology. 2014;31(10):191.

16. Middleton G, Silcocks P, Cox T, Valle J, Wadsley J, Propper D, et al. Gemcitabine and capecitabine with or without telomerase peptide vaccine GV1001 in patients with locally advanced or metastatic pancreatic cancer (TeloVac): an open-label, randomised, phase 3 trial. The Lancet Oncology. 2014;15(8):829-40. 
17. Shima H, Kutomi G, Satomi F, Imamura M, Kimura Y, Mizuguchi T, et al. Case report: Long-term survival of a pancreatic cancer patient immunized with an SVN-2B peptide vaccine. Cancer immunology, immunotherapy : CII. 2018;67(10):1603-9.

18. Suzuki N, Hazama S, Iguchi H, Uesugi K, Tanaka H, Hirakawa K, et al. Phase II clinical trial of peptide cocktail therapy for patients with advanced pancreatic cancer: VENUS-PC study. Cancer science. 2017;108(1):73-80.

19. Weden S, Klemp M, Gladhaug IP, Moller M, Eriksen JA, Gaudernack G, et al. Long-term follow-up of patients with resected pancreatic cancer following vaccination against mutant K-ras. Int $\mathrm{J}$ Cancer. 2011;128(5):1120-8.

20. Gjertsen MK, Buanes T, Rosseland AR, Bakka A, Gladhaug I, Soreide O, et al. Intradermal ras peptide vaccination with granulocyte-macrophage colony-stimulating factor as adjuvant: Clinical and immunological responses in patients with pancreatic adenocarcinoma. Int J Cancer. 2001;92(3):44150 .

21. Kirner A, Mayer-Mokler A, Reinhardt C. IMA901: a multi-peptide cancer vaccine for treatment of renal cell cancer. Hum Vaccin Immunother. 2014;10(11):3179-89.

22. Bais P, Namburi S, Gatti DM, Zhang X, Chuang JH. CloudNeo: a cloud pipeline for identifying patientspecific tumor neoantigens. Bioinformatics. 2017;33(19):3110-2.

23. Hundal J, Miller CA, Griffith M, Griffith OL, Walker J, Kiwala S, et al. Cancer Immunogenomics: Computational Neoantigen Identification and Vaccine Design. Cold Spring Harb Symp Quant Biol. 2016;81:105-11.

24. Schenck RO, Lakatos E, Gatenbee C, Graham TA, Anderson ARA. NeoPredPipe: high-throughput neoantigen prediction and recognition potential pipeline. BMC Bioinformatics. 2019;20(1):264.

25. Tappeiner E, Finotello F, Charoentong P, Mayer C, Rieder D, Trajanoski Z. TIminer: NGS data mining pipeline for cancer immunology and immunotherapy. Bioinformatics. 2017;33(19):3140-1.

26. Torres-Garcia W, Zheng S, Sivachenko A, Vegesna R, Wang Q, Yao R, et al. PRADA: pipeline for RNA sequencing data analysis. Bioinformatics. 2014;30(15):2224-6.

27. Ryan DP, Hong TS, Bardeesy N. Pancreatic adenocarcinoma. The New England journal of medicine. 2014;371(22):2140-1.

28. Conroy T, Desseigne F, Ychou M, Bouche O, Guimbaud R, Becouarn Y, et al. FOLFIRINOX versus gemcitabine for metastatic pancreatic cancer. The New England journal of medicine. 2011;364(19):1817-25.

29. Von Hoff DD, Ervin T, Arena FP, Chiorean EG, Infante J, Moore M, et al. Increased survival in pancreatic cancer with nab-paclitaxel plus gemcitabine. The New England journal of medicine. 2013;369(18):1691-703.

30. Vienot A, Beinse G, Louvet C, de Mestier L, Meurisse A, Fein F, et al. Overall Survival Prediction and Usefulness of Second-Line Chemotherapy in Advanced Pancreatic Adenocarcinoma. Journal of the National Cancer Institute. 2017;109(10). 
31. Rahma OE, Hamilton JM, Wojtowicz M, Dakheel O, Bernstein S, Liewehr DJ, et al. The immunological and clinical effects of mutated ras peptide vaccine in combination with IL-2, GM-CSF, or both in patients with solid tumors. Journal of translational medicine. 2014;12:55.

\section{Tables}

Table 1. Baseline characteristics of included patients

\begin{tabular}{|c|c|c|}
\hline \multirow[t]{2}{*}{ Characteristics } & \multicolumn{2}{|c|}{ Patient $(\mathrm{N}=7)$} \\
\hline & $\mathrm{n}$ & $\%$ \\
\hline \multicolumn{3}{|l|}{ Sex } \\
\hline Male & 5 & 71.43 \\
\hline Female & 2 & 28.57 \\
\hline \multicolumn{3}{|l|}{ Age } \\
\hline$<60$ & 3 & 42.86 \\
\hline$>=60$ & 4 & 57.14 \\
\hline \multicolumn{3}{|l|}{ Tumor location } \\
\hline Pancreatic head & 2 & 28.57 \\
\hline Pancreatic body and tail & 5 & 71.43 \\
\hline \multicolumn{3}{|l|}{ Histopathology } \\
\hline Adenocarcinoma & 6 & 85.71 \\
\hline Others & 1 & 14.29 \\
\hline \multicolumn{3}{|l|}{ Metastatic sites } \\
\hline Liver & 4 & 57.14 \\
\hline Peritoneum & 3 & 42.86 \\
\hline \multicolumn{3}{|l|}{ ECOG score } \\
\hline 0 & 3 & 42.86 \\
\hline 1 & 4 & 57.14 \\
\hline \multicolumn{3}{|l|}{ CA19-9 level(first visit) } \\
\hline$<37.0$ & 3 & 42.86 \\
\hline$>=37.0$ & 4 & 57.14 \\
\hline
\end{tabular}


ECOG, Eastern cooperative oncology group; CA19-9, Carbohydrate antigen 19-9;

Table 2. Comparison of adverse reactions between peptide therapy and chemotherapy in treated patients

\begin{tabular}{|lllll|}
\hline Adverse Effects & Any grade & & Grades 3 to 4 & \\
\cline { 2 - 5 } & Chemotherapy & $\begin{array}{l}\text { Neoantigen } \\
\text { vaccine }\end{array}$ & Chemotherapy & $\begin{array}{l}\text { Neoantigen } \\
\text { vaccine }\end{array}$ \\
\cline { 2 - 5 } & $\mathrm{N}(\%)$ & $\mathrm{N}(\%)$ & $\mathrm{N}(\%)$ & $\mathrm{N}(\%)$ \\
\hline Gastrointestinal reaction & $7(100.0)$ & $0(0.0)$ & $2(28.6)$ & $0(0.0)$ \\
\hline Thrombocytopenia & $5(71.4)$ & $0(0.0)$ & $2(28.6)$ & $0(0.0)$ \\
\hline Rash & $2(28.6)$ & $1(14.3)$ & $0(0.0)$ & $0(0.0)$ \\
\hline Fever & $1(14.3)$ & $0(0.0)$ & $0(0.0)$ & $0(0.0)$ \\
\hline Neutropenia & $7(100.0)$ & $0(0.0)$ & $4(57.1)$ & $0(0.0)$ \\
\hline $\begin{array}{l}\text { Peripheral nerve } \\
\text { abnormalities }\end{array}$ & $3(42.9)$ & $0(0.0)$ & $0(0.0)$ & $0(0.0)$ \\
\hline Anemia & $5(71.4)$ & $0(0.0)$ & $1(14.3)$ & $0(0.0)$ \\
\hline Elevated transaminase & $3(42.9)$ & $0(0.0)$ & $0(0.0)$ & $0(0.0)$ \\
\hline Fatigue & $7(100.0)$ & $0(0.0)$ & $1(14.3)$ & $0(0.0)$ \\
\hline
\end{tabular}

Table 3. The survival and objective response rate of patients 


\begin{tabular}{|ll|}
\hline Survival & Months \\
\hline mOS & 24.1 \\
\hline mPFS & 3.1 \\
\hline mOS* & 8.3 \\
\hline Objective response rate & $\mathrm{n} / \mathbf{N}$ \\
\hline CR & $0 / 7$ \\
\hline PR & $2 / 7$ \\
\hline SD & $4 / 7$ \\
\hline PD & $1 / 7$ \\
\hline
\end{tabular}

mOS, mean Overall survival; mPFS, mean Progression-free survival; CR, Complete remission; PR, Partial response; SD, Stable disease; PD, Progressive disease;

* Calculated from the time the patient received the peptide vaccine

Figures

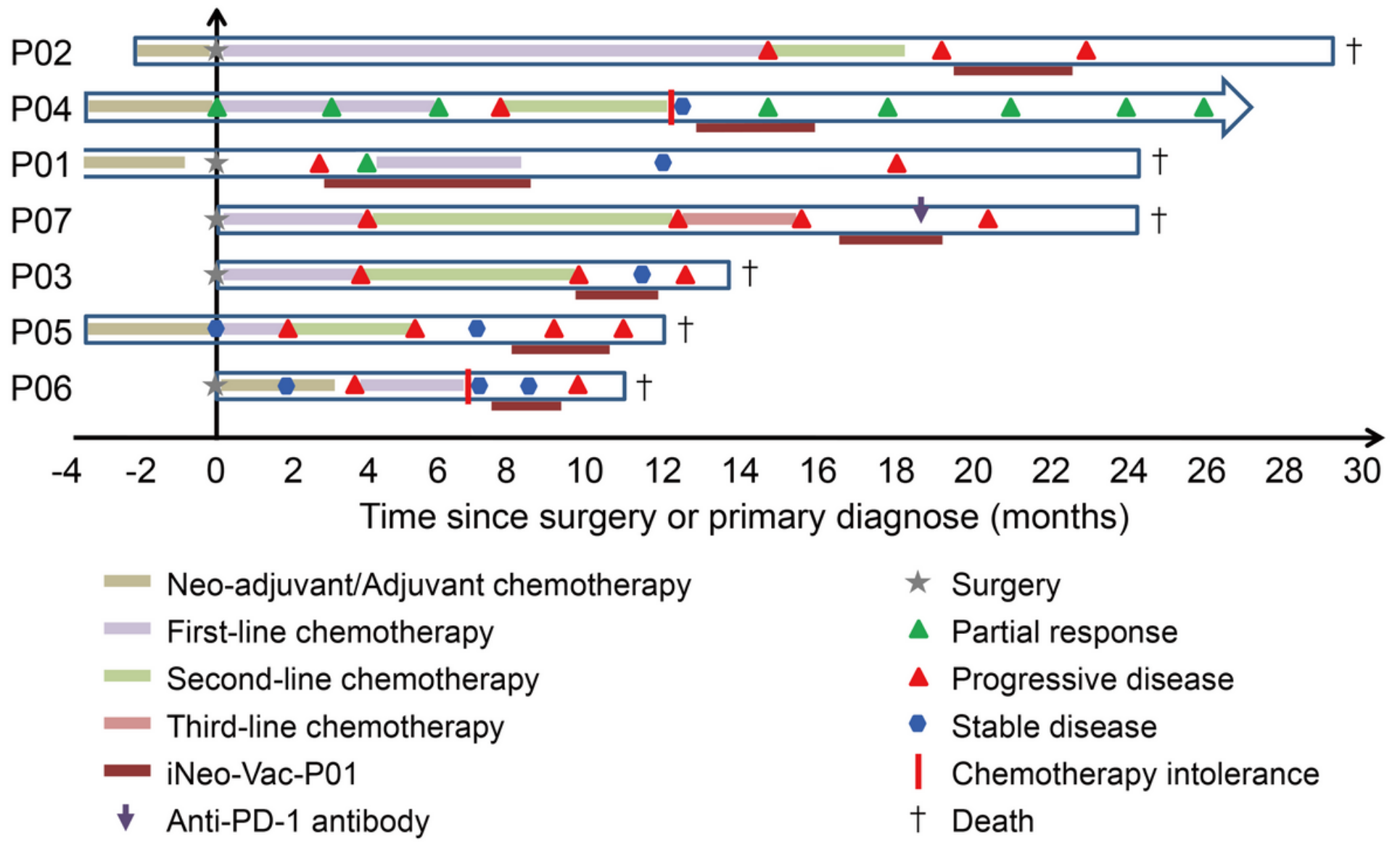

Figure 1 
Clinical treatment process for seven vaccinated patients from surgery or primary visit until the end of follow-up.

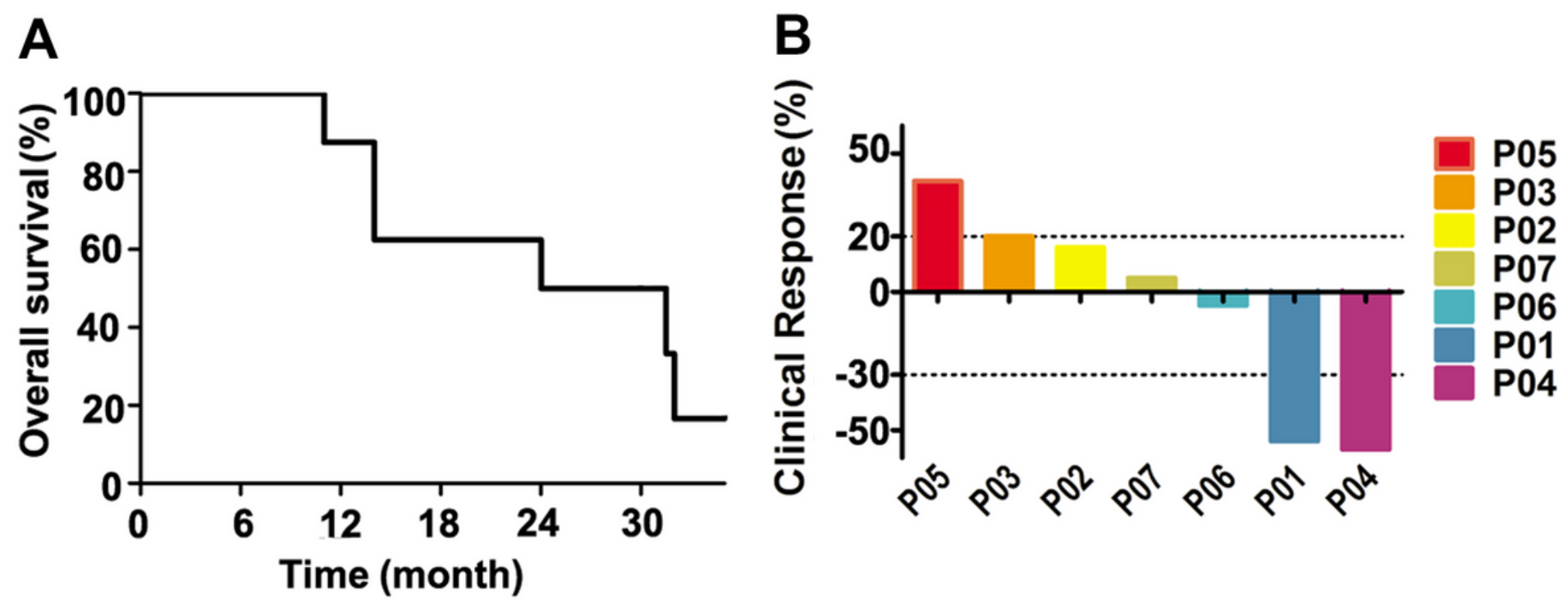

Figure 2

The clinical response and prognosis of treated patients. A. The overall survival curve of seven patients. B. The percentage changes of tumor lesion size from baseline. The changes in lesions between a positive value of $25 \%$ and a negative value of $50 \%$ are considered stable disease. 
A

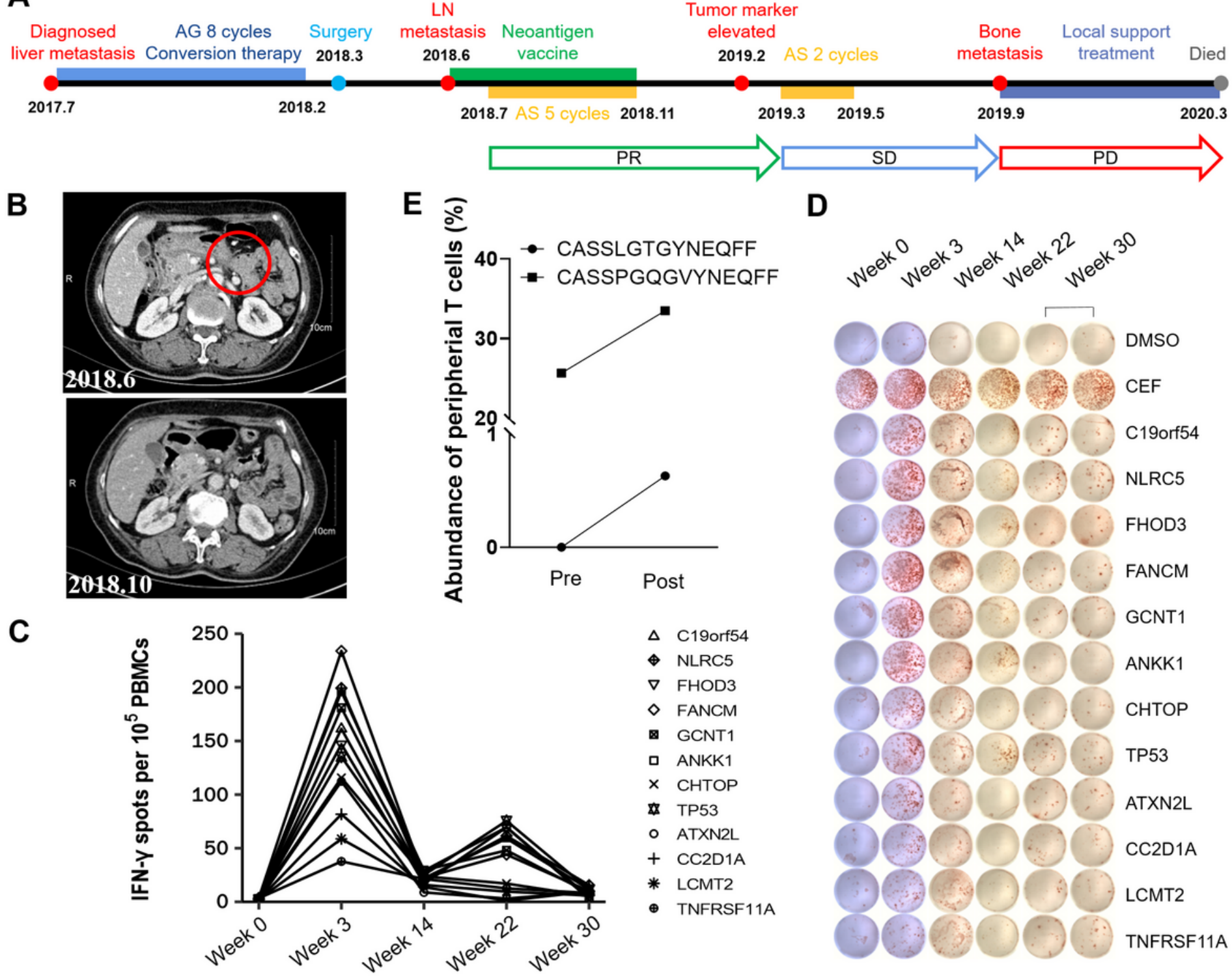

Figure 3

A case report of Patient P01. A. Treatment timeline of P01. B. The imaging comparison of lymph node changes during neonatal antigen treatment, efficacy evaluation was PR. C and D. Ex vivo IFN- $\gamma$ ELISpot of PBMCs was performed with peptides at different time points. The dimethyl sulfoxide (DMSO) group was used as the negative control and mixed peptides from CEF (including peptides of cytomegalovirus, epstein-barr virus and influenza virus) were used as the positive control. E. Increased abundance of peripheral T cell clones after vaccination was detected by TCR sequencing. 


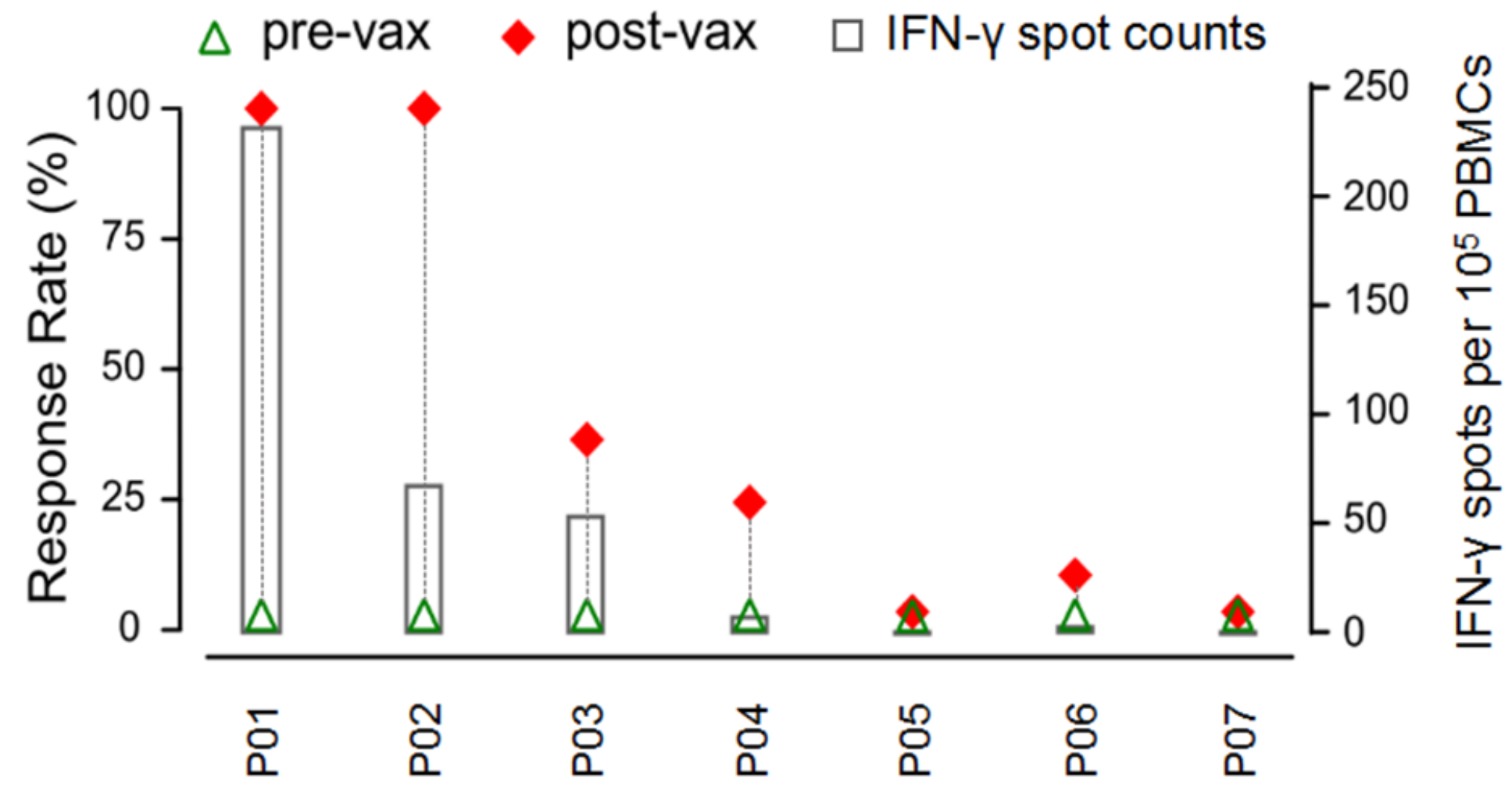

Figure 4

iNeo-Vac-P01 induced specific T cell response. For each patient marked in X-axis, green triangle and red diamond represent the response rates pre- and post-vaccination, defined as the ratios of the numbers of peptides (or peptide pools) with positive ELISpot results before and after vaccination to the total number of peptides (or peptide pools) in vaccine, respectively. The bar chart with secondary Y-axis represented the IFN-y spots per 105 PBMCs of the peptide with best response for each patient. 


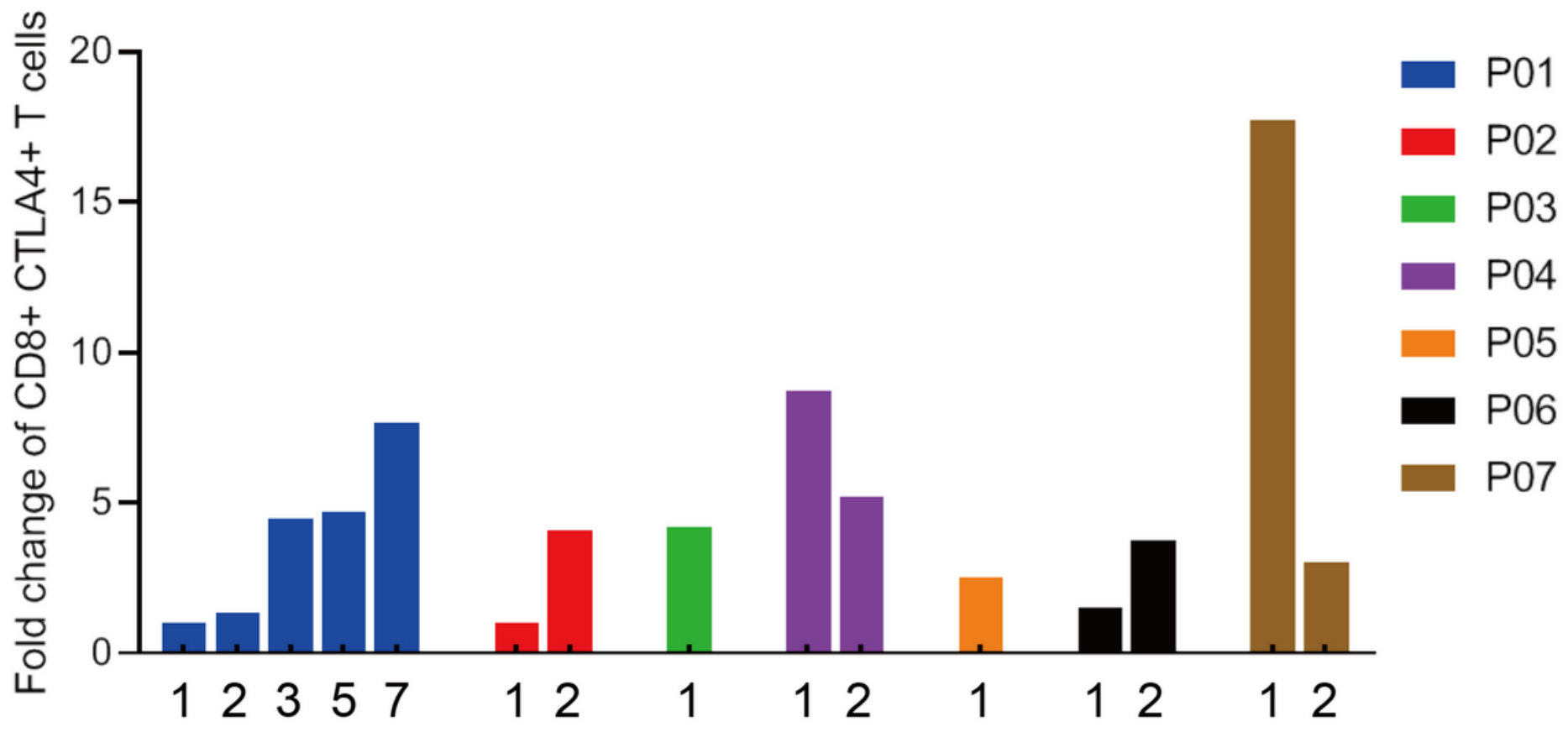

Time after vaccination (month)

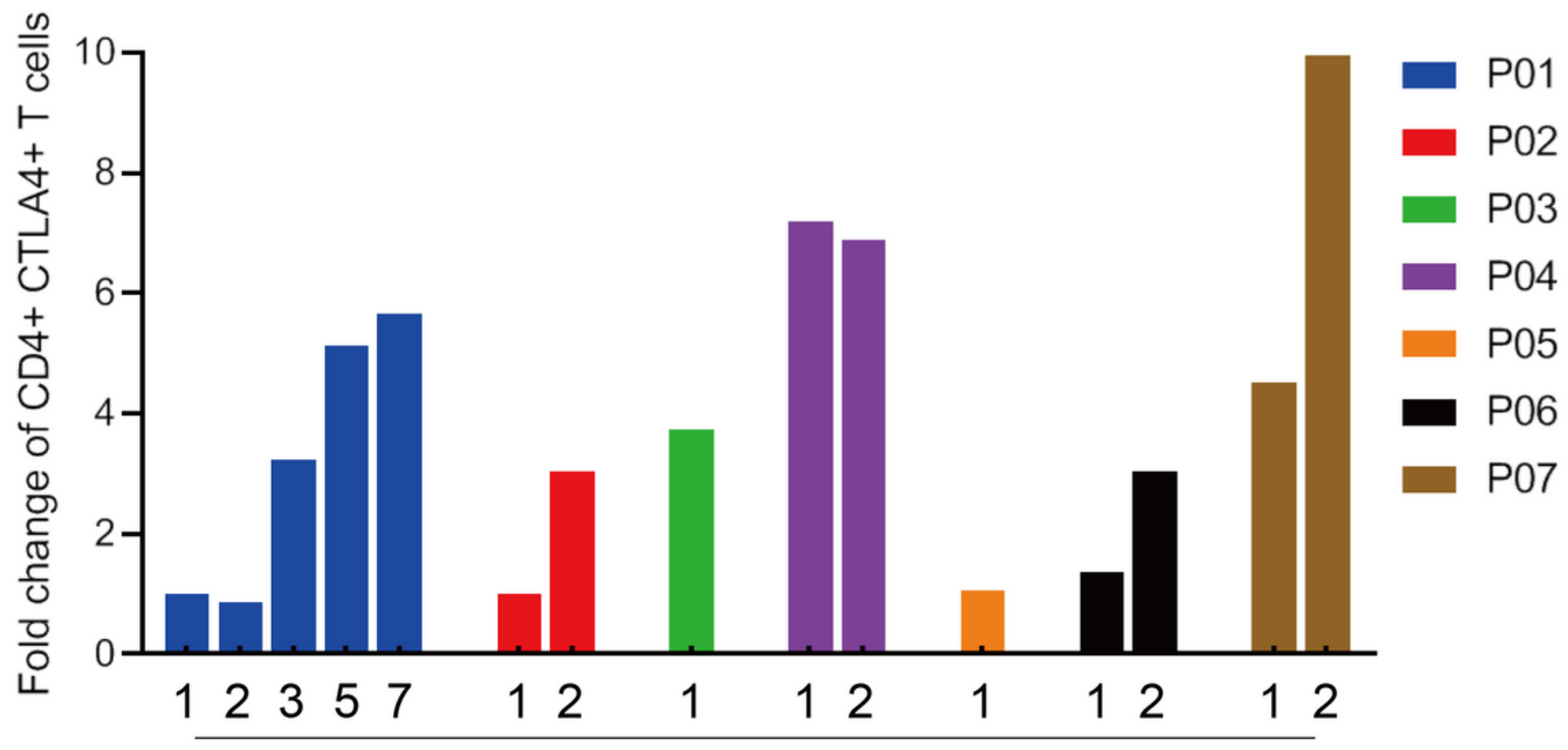

Time after vaccination (month)

Figure 5

The proportion of CTLA4+ T cells in peripheral blood increased after vaccination. The proportions of CD8+ CTLA-4+ T cells and CD4+ CTLA-4+ T cells ratio to total T cells in peripheral blood were detected by flow cytometry. Fold changes of post vaccination compared with baseline were calculated.

\section{Supplementary Files}


This is a list of supplementary files associated with this preprint. Click to download.

- renamed19d0d.xlsx

- renamedd4ba9.docx 